\title{
Network Slicing to Enable Resilience and High Availability in 5G Mobile Telecommunications
}

\author{
Qi He${ }^{1}$, Yunxia $\mathrm{Ju}^{3}$,Jianguo Wang ${ }^{1}$, Gang Zhao ${ }^{1}$, Haiyong Qin ${ }^{1}$, Kai Zhao ${ }^{4}$,Yilan Zhou ${ }^{1}$ MinLi $^{1}$,Qi Dong ${ }^{1}$ \\ ${ }^{1}$ Shool of mathematical sciences, Qilu Normal University,Jinan 250200, China. \\ ${ }^{2}$ School of Information Science and Engineering, Qilu Normal University, Jinan 250200, China. \\ ${ }^{3}$ School of Information Science and Engineering, Shandong Normal University, Jinan 250014, China. \\ ${ }^{4}$ School of Electrical Engineering and Automation, Qilu University of Technology(Shandong Academy of Sciences), Jinan 250353, China.
}

\begin{abstract}
In the upcoming fifth-generation (5G) ecosystem, the delivery of a variety of personalized services is envisioned. With the development of software-defined networks and network function virtualization technologies, networks display increasingly flexible features, such as programmability. Network slicing is a state-of-the-art technology that provides services tailored to the specific demands of users, such as smart grids and e-health applications. In this article, we introduce the network slicing concept and its application and discuss related work. In addition, we propose an architecture for network slicing by combining software-defined networks and network function virtualization technologies. Finally, we note important challenges and open issues in the development and application of these technologies.
\end{abstract}

\section{INTRODUCTION}

Currently, diverse mobile terminal devices are found almost everywhere, and mobile data traffic is growing rapidly. The increasing adoption of these technologies has driven the development of the next generation of networks. Fifth-generation mobile technology $(5 \mathrm{G})$ is intended to satisfy service requirements with respect to supporting multiple types of devices and user interactions [1]. In the future, $5 \mathrm{G}$ will support a variety of applications, such as delay-sensitive video, smart grids and e-health applications.

Traditional one-size-fits-all networks are unable to meet various requirements in terms of reliability, latency, and flexibility. 5G networks are intended to meet all types of user requirements in different application scenarios and to reduce capital and operational expenditures compared with today's environments. $5 \mathrm{G}$ systems should provide users with services anytime and anywhere, even on high-speed trains at speeds above 500 kilometers per hour or aircraft at altitudes of up to $12 \mathrm{~km}$. In many urban areas, population densities of thousands of people per square kilometer are expected to be common. In extreme real-time communications, autonomous driving requires ultrareliable communications with strongly reduced latency to permit the immediate reactions needed to prevent road accidents. Furthermore, next-generation mobile networks should be able to provide reliable communication services in the case of public safety announcements or natural disasters, such as floods and earthquakes. All types of basic communication, such as video and audio, are needed in disaster areas. Survivors can use these forms of communication to request rescue. In an emergency, the energy consumption of the network and the user devices is crucial. Table I shows the requirements of different use case categories.

Table IRequirements of different use case categories [1]

\begin{tabular}{|c|c|c|c|c|c|}
\hline $\begin{array}{c}\text { Use case } \\
\text { category }\end{array}$ & UEDR & $\begin{array}{c}\text { E2E } \\
\text { latency }\end{array}$ & Mobility & $\begin{array}{c}\text { Connection } \\
\text { density }\end{array}$ & $\begin{array}{c}\text { Traffic } \\
\text { density }\end{array}$ \\
\hline $\begin{array}{c}\text { Broadband } \\
\text { access in densely } \\
\text { populated areas }\end{array}$ & $\begin{array}{c}\text { DL: } 300 \\
\text { Mbps UL:50 } \\
\text { Mbps }\end{array}$ & $10 \mathrm{~ms}$ & $\begin{array}{c}\text { On demand, 0-100 } \\
\mathrm{km} / \mathrm{h}\end{array}$ & $\begin{array}{c}200-2500 / \mathrm{km} \\
2\end{array}$ & $\begin{array}{c}\text { DL: } 750 \\
\mathrm{Gbps} / \mathrm{km}^{2} \mathrm{UL}: \\
125 \mathrm{Gbps} / \mathrm{km}^{2}\end{array}$ \\
\hline $\begin{array}{c}\text { Mobile } \\
\text { broadband in } \\
\text { vehicles }\end{array}$ & $\begin{array}{c}\text { DL:50 Mbps } \\
\text { UL:25 Mbps }\end{array}$ & $10 \mathrm{~ms}$ & $\begin{array}{c}\text { On demand, up to100 } \\
\mathrm{km} / \mathrm{h}\end{array}$ & $2000 / \mathrm{km}^{2}$ & $\begin{array}{c}\mathrm{DL}: 100 \\
\mathrm{Gbps} / \mathrm{km}^{2} \mathrm{UL}: \\
50 \mathrm{Gbps} / \mathrm{km}^{2}\end{array}$ \\
\hline $\begin{array}{c}\text { Ultralow latency } \\
\text { DL:50 Mbps } \\
\text { UL:25 Mbps }\end{array}$ & $<1 \mathrm{~ms}$ & Pedestrian & Not critical & Potentially \\
\hline $\begin{array}{c}\text { Indoor } \\
\text { ultra-high-speed } \\
\text { broadband }\end{array}$ & $\begin{array}{c}\text { DL: } 1 \mathrm{Gbps} \\
\mathrm{UL}: 500 \\
\mathrm{Mbps}\end{array}$ & $10 \mathrm{~ms}$ & Pedestrian & $75,000 / \mathrm{km}^{2}$ & $\begin{array}{c}\text { DL: } 15 \\
\mathrm{Tbps} / \mathrm{km}^{2} \mathrm{UL}: \\
2 \mathrm{Tbps} / \mathrm{km}^{2}\end{array}$ \\
\hline
\end{tabular}




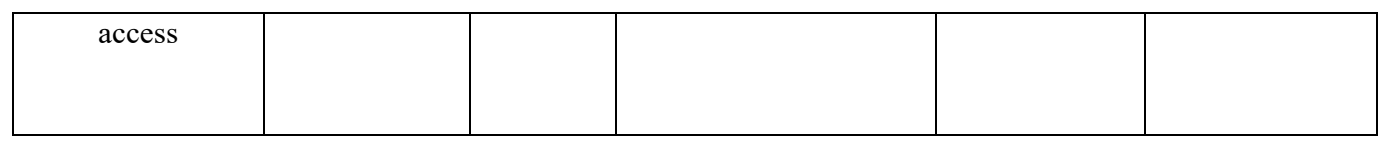

UEDR: user-experienced data rate, DL: downlink, UL: uplink

Given the personalized and customized service requirements mentioned above, the concept of network slicing has been proposed. In the following sections, we will introduce the concept of network slicing.

\subsection{Definition of Network Slices}

Network slices are end-to-end logical networks that run on a common underlying physical network to support on-demand customized services with independent control and management. By dividing the physical network into multiple dedicated virtual mobile networks with varying levels, network slicing can provide customized services for different application scenarios [2]-[4]. The concept of network slicing is shown in Fig. 1.

Network slicing is a promising technology and one of the most important concepts for $5 \mathrm{G}$ networks, which provides some services tailored to the specific demands of users. Network slicing defines logical networks that include both shared and dedicated resources, such as virtual network functions, radio spectrum bands or network equipment.

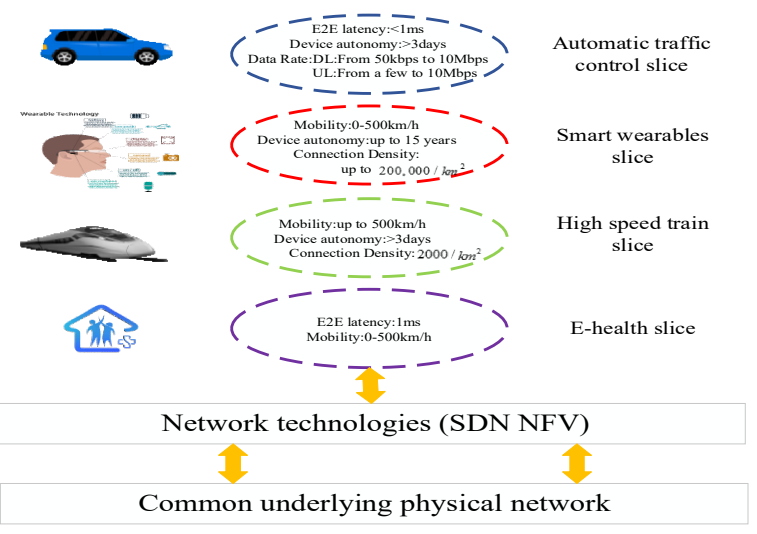

Fig. 1. The concept of network slicing

The software-defined networking (SDN) architecture is provided by the Open Network Foundation (ONF). In this architecture, the data and control planes are decoupled. Carriers gain unprecedented network control and programmability. The OpenFlow protocol, which provides high-performance traffic control, is a critical element of the SDN architecture [5]. Network function virtualization (NFV) implements network functions through software virtualization [6]. The European Telecommunications Standards Institute (ETSI) has studied the integration of SDN into NFV architecture. Through SDN and NFV technologies, network softwarization can provide resilience, scalability and high availability. In the next-generation mobile network architecture, the control plane and the user plane should be separated, in accordance with SDN principles.

\subsection{Network Slicing Applications}

With unified physical infrastructures, network slicing is able to meet diverse network requirements. Slicing applications include smart wearables, smart offices, automated traffic control and driving, remote computing, and e-health applications.

(1)Smart wearables include multiple types of small devices and microsensors. Some low-power, ultralight sensors will be integrated into clothes, shoes or hats. These sensors can measure a variety of physiological parameters, such as blood pressure, heart rate, and temperature. The key challenges in these applications include the efficient management of a variety of devices and the large amounts of data that are produced.

(2) In smart offices, it is expected that the vast majority of devices will be connected wirelessly. Communication among personnel will be carried out through various wireless devices. Thus, hundreds of users need ultrahigh bandwidth to provide intensive communications and video instant messaging services. The aggregation of all of these services leads to a very high density of devices, which have very different characteristics and are expected to be combined in an interacting framework.

(3) Automated driving will require increased data rates between vehicles and the supporting infrastructure, as well as ultralow latency and ultrahigh reliability to provide warning signals. These applications will communicate with users, including cyclists and pedestrians. Remote computing will be used at high speeds (e.g., on public transportation). The automotive industry will rely on remote processing. All of these applications will require robust communication.

(4) With the development of informationization, remote health monitoring will bring convenience to people's lives. Users will employ various electronic devices to collect various body parameters. Using mobile devices, doctors will view relevant information and send their opinions to users in the form of information. This framework will greatly reduce costs and improve efficiency.

\subsection{Related Work}

In 2017, Huawei released a network slicing white paper [7]. Ericsson and two other companies are also working on network slicing [8]. Learning from previous work on network slicing and elaborating the principles and architectures used in network slicing from different perspectives is valuable.

In [2], the authors propose a high-level architectural solution and discuss the need for different levels of mobile network customization. They assess the potential of network slicing and highlight the technology challenges. Article [4] elaborates the concept of network slicing and provides an overview of SDN architecture. Moreover, the article presents a scenario to realize network slices. In [9], 
a data-driven architecture is proposed for $5 \mathrm{G}$ networks, which enhances the quality of experience (QoE) for individual users. Under this architecture, authors propose a two-step QoE modeling approach. Another paper [6] explores the potential of network function virtualization (NFV) in enhancing the functional, architectural and commercial viability of $5 \mathrm{G}$ radio access networks. The article describes NFV implementation in SDN and network overlay technologies. Paper [10] analyzes the radio access network (RAN) slicing problem and presents four different RAN slicing approaches. The authors of [11] propose an information-centric networking architecture and introduce a general application-driven framework that emphasizes the flexibility afforded by NFV and SDN and over which 5G-ICN (information-centric networking) can be realized.

In this article, the fundamental concept of network slicing is introduced. This paper also introduces the application of network slicing and related work on network slicing. The remainder of this paper is arranged as follows. Section 2 describes the network slicing architecture, and network slicing management is described. Several open issues and challenges are discussed in section 3. Finally, section 4 provides the conclusion.

\section{Network slicing architecture-based 5G mobile communication}

Traditional network architectures are poorly suited to meet the requirements of today's companies, operators, and end users. The design of the network slicing architecture in 5G mobile communications should be based on comprehensive considerations. The architectural design should be based on the following principles: (1) Configuration and self-healing are automated. (2) The numbers of functionalities and entities are minimized. (3) Fixed and mobile convergence is supported. (4) Network reliability and security are enhanced.

In this section, network slicing architecture-based $5 \mathrm{G}$ mobile communication is shown in Fig. 2. In the designed architecture, a three-layer structure is proposed that incorporates an infrastructure layer (IL), a network function layer (NFL), and a business layer (BL). In [12], an architecture design scheme is proposed. However, this article proposes to increase the content of the IL. As network slicing is a novel concept, the proposed architecture can solve many important design problems related to network hierarchical management. In the following, we detail each layer of the presented architecture.

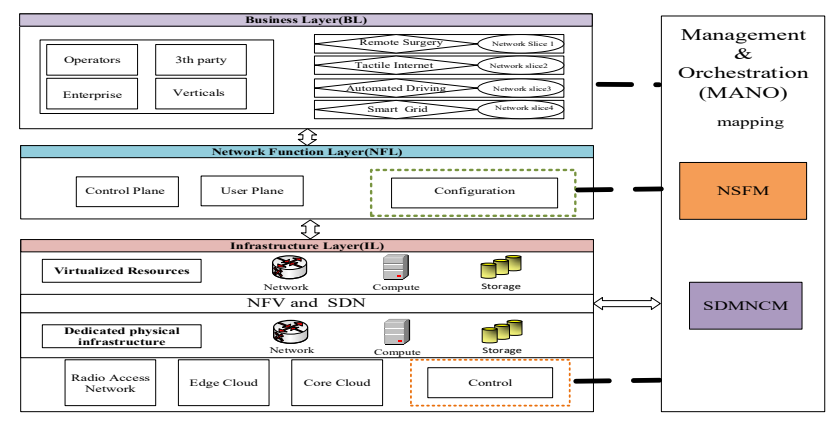

NSFM: Network Slice Function Manager SDMNCM: software-defined mobile network control and management

Fig. 2. Network slicing architecture.

\subsection{Infrastructure Layer (IL)}

This layer consists of physical infrastructure, virtualization resources, NFV and SDN, and it will support connectivity as a service. The IL includes the deployment, control and management of the infrastructure. Using SDN, NFV and other related technologies, physical network resources, including computing, storage, and network, are converted into virtual network resources. Furthermore, different infrastructural elements can be used to meet the needs of a variety of slices.

Given the core network and the edge network, there is general agreement to deploy virtual network functionality using the general hardware infrastructure. However, because of the different constraints of the various services deployed on the network, a simple centralized positioning cloud infrastructure may not be suitable for all slices. This layer also includes edge cloud and core cloud infrastructures, and efficient SDN control methodologies are used to promote network agility.

\subsection{Network Function Layer (NFL)}

The NFL includes the user plane and the control plane, which can be configured through a unified manager. This layer can be adapted to specific use cases, transforming network slices into resources and service operational packages. All of the functions associated with slicing are performed in this layer. This layer supports management of the life cycle, including management, control, adjustment and termination, for each network slice. It also provides interfaces across the network applications and functions. Network services run under the control and supervision of a network slice function manager (NSFM).

\subsection{Business Layer (BL)}

Networks must be able to satisfy a wide range of requirements, including remote surgery, tactile Internet, automated driving and smart grids. The 5GMF white paper divides the communication of the $5 \mathrm{G}$ mobile network into three categories: enhanced mobile broadband (eMBB), large machine-type communication (mMTC) and ultrareliable and low-latency communication (URLLC). 


\subsection{Orchestration and Management}

Efficient orchestration and management functions are essential components of mobile networks. Each slice must be independently managed and have independent security capabilities that prevent unauthorized entities from accessing management information. SDN functions have recently been developed to perform network operations. The control functions are separated from the data plane through a low-complexity interface. This decoupling provides greater flexibility, scalability, and availability while reducing costs. For specific services, the behavior of networks can be changed by simply modifying the software that controls functions. The control of the $5 \mathrm{G}$ mobile network architecture adopts the software-defined mobile network control and management (SDMNCM) concept. Functions are executed by the controller, including the functions of the mobile network control plane. We suggest developing a logical information base that can be used in SDMNCM. Data can be placed arbitrarily on the edge cloud. Using a software-defined method, all of these functions can be executed by a programmable software-defined mobile controller, which would be of great value in the operation of mobile networks.

\subsection{The Key Advantages of the Proposed Architecture}

(1) High availability and resilience. High availability and resilience are essential in ensuring that minimum service is provided when disasters occur [1]. Resilience, the ability to recover from failures, is an important feature of a network. The problem faced by network operators is that wireless devices are very expensive, which does not suit their needs. Using the proposed approach, the operator can reduce costs by simply reprogramming the controller to adapt the equipment to their needs while reducing costs and enhancing reliability.

(2) Flexibility and scalability. the system design splits the network domains according to their functions. The RAN and core network domains are functionally decoupled. To provide the utilization efficiency of network resources, this system can dynamically relocate network resources depending on current needs.

(3) Programmability. The network control plane is decoupled. Network operators can programmatically configure, manage and optimize this type of network. This capability can significantly improve performance and optimize resource allocation.

To implement slicing, we need to adopt the infrastructure as a service (iaas) paradigm, in which different infrastructural elements can be rented to accommodate different requirements. We consider the operation of a multicloud provider, where both physical and virtual infrastructure can be provided as services (i.e., monitoring as a service, MaaS).

\section{CHALLENGES AND OPEN ISSUES}

For next-generation mobile networks, network slicing is a very promising model, but its realization involves certain challenges. following.

\subsection{End-to-End Slice Management}

An important challenge is how to realize a network slice from a high-level description of services to the infrastructure and network function of the specific section. One good way to address this issue is to develop and use a specific description language. The key features of the language itself are as follows. The language should provide extensibility, the flexibility required to adapt to possible future network elements, and the applicability of the environment by a number of suppliers.

\subsection{Security Concerns}

Security is a basic necessity in handling vast amounts of data that must be protected against attacks. Programmable open interfaces pose a potential threat to software networks. This threat requires a consistent multilevel security framework consisting of policies for the remote authentication of software integrity. The security problems caused by $5 \mathrm{G}$ slicing are the major barrier to adopting multitenancy today. The robustness of the system and the network resilience must be improved. Faults occurring in one slice must not have an effect on other slices.

\subsection{Operational Efficiency}

Operational efficiency is a key factor in reducing network costs. Self-management functions should support a flexible architecture to cope with different use cases. Management interfaces can be implemented using a novel paradigm with the support of the open-source community. This management framework allows partners to exchange management information. Service management processes rely on autonomous functions, self-configuration, and self-healing.

\section{CONCLUSION}

Network slices are a novel means of realizing the potential of $5 \mathrm{G}$ networks. In this paper, we discuss the concept of network slicing, its applications and the integration of other technologies. We propose a network slicing architecture-based $5 \mathrm{G}$ ecosystem. Finally, we discuss the remaining challenges and open issues. As SDN and NFV technologies mature, operators will provide increasingly customized services to the vertical industry in a flexible way. We firmly believe that network slicing will play an important role in $5 \mathrm{G}$ network.

\section{Acknowledgment}

Shandong Provincial Natural Science Foundation (Grant No. ZR2016AB04) 
a Project of Shandong Province Higher Educational Science and Technology Program(Grant no. J17KB121))

\section{REFERENCES}

1. R. El Hattachi and J. Erfanian, NGMN $5 G$ White Paper. Next generation Mobile (NGMN) Ltd.,(2015)

2. T. Taleb, B. Mada, M. I. Corici, A. Nakao, and H. Flinck, "PERMIT: Network slicing for personalized 5G mobile telecommunications," IEEE Commun. Mag., vol. 55, no. 5, pp. 88-93, May (2017)

3. K. Katsalis, N. Nikaein, E. Schiller, A. Ksentini, and T. Braun, "Network slices toward $5 \mathrm{G}$ communications: Slicing the LTE network," IEEE Commun. Mag., vol. 55, no. 8, pp. 146-154, Aug. (2017)

4. J. Ordonez-Lucena, P. Ameigeiras, D. Lopez, J. J. Ramos-Munoz, J. Lorca, and J. Folgueira, "Network slicing for $5 \mathrm{G}$ with SDN/NFV: Concepts, architectures, and challenges," IEEE Commun. Mag., vol. 55, no. 5, pp. 80-87, May (2017)

5. Open Networking Foundation, Software-Defined Networking:The New Norm for Networks. White Paper.

6. S. Abdelwahab, B. Hamdaoui, M. Guizani, and T. Znati, "Network function virtualization in $5 \mathrm{G}$," IEEE Commun. Mag., vol. 54, no. 4, pp. 84-91, Apr. (2016)

7. White Paper, 5G Service Guaranteed Network Slicing White Paper, (2017)

8. Ericsson study:How network slicing pays off. Available:

https://www.ericsson.com/en/press-releases/ (2017)

9. Y. Wang, P. Li, L. Jiao, Z. Su, N. Cheng, X. S. Shen, and P. Zhang, "A data-driven architecture for personalized QoE management in 5G wireless networks," IEEE Wireless Commun., vol. 24, no. 1, pp. 102-110, Nov. (2017)

10. O. Sallent, J. Perez-Romero, R. Ferrus, and R. Agusti, "On radio access network slicing from a radio resource management perspective," IEEE Wireless Commun., vol. 24, no. 5, pp. 166-174, Apr. (2017)

11. R. Ravindran, A. Chakraborti, S. O. Amin, A. Azgin, and G. Wang, "5G-ICN: Delivering ICN services over 5G using network slicing," IEEE Commun. Mag., vol. 55, no. 5, pp. 101-107, (2017)

12. X. Foukas, G. Patounas, A. Elmokashfi, and M. K. Marina, "Network slicing in 5G: Survey and challenges," IEEE Commun. Mag., vol. 55, no. 5, pp. 94-100, (2017) 